\title{
Chemical reaction between hydrogen atoms and electrons on the surface of superfluid ${ }^{4} \mathrm{He}$
}

\author{
T. Arai ${ }^{a, *}$, A. Würl ${ }^{b}$, P. Leiderer ${ }^{b}$, T. Shiino ${ }^{a}$, K. Kono ${ }^{a}$ \\ "Institute for Solid State Physics, University of Tokyo, 7-22-1, Roppongi, Minato-ku, Tokyo, 106-8666. Japan

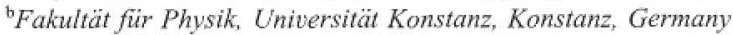

\section{Abstract}

Two-dimensional mixture of atomic hydrogen and electrons on the surface of superfluid ${ }^{4} \mathrm{He}$ was realized below $0.5 \mathrm{~K}$. Vibrating capacitor electrometer was employed to show that after the electron capture reaction, $\mathrm{H}^{+} \mathrm{e}^{-} \rightarrow \mathrm{H}^{-}$, the product, $\mathrm{H}^{-}$, might penetrate into the bulk liquid.

Keywords: Electron capture; Hydrogen; Surface electrons; Vibrating capacitor electrometer

In our recent report, we showed that in the two-dimensional mixture of hydrogen atoms $(\mathrm{H})$ and electrons $\left(\mathrm{e}^{-}\right)$on the surface of superfluid ${ }^{4} \mathrm{He}$, a chemical reaction takes place to destroy the surface state electrons [1]. We believe that it is due to the electron capture reaction by $\mathrm{H}, \mathrm{H}+\mathrm{e}^{-} \rightarrow \mathrm{H}^{-}+0.75 \mathrm{eV}$.

We are interested in the question whether $\mathrm{H}^{-}$stays on the surface or penetrates into the bulk liquid. We could not give the answer to the question because the sensor employed in the previous experiment was Corbino electrode, which is suitable for conductivity measurements of the surface charge. Even if $\mathrm{H}^{-}$were created and it stayed on the surface, its contribution would be hidden under the much larger conductivity of surface electrons.

In this work, we developed the vibrating capacitor electrometer (VCE) technique to measure the total charge on the surface. If $\mathrm{H}^{-}$stays on the surface, the total charge will not be influenced by the electron capture reaction or vice versa.

A pair of parallel plate electrodes are placed in the sample cell and liquid helium is filled up to the midway between the electrodes. A positive DC voltage $\phi$ is applied to the bottom electrode to hold the surface electrons. The electric field above the liquid is partly

*Corresponding author. Tel: + 817-3-3478-6811 ex. 5221; fax: $+817-3-3478-6843$.

E-mail address: toshikaz@issp.u-tokyo.ac.jp (T. Arai) screened by the surface negative charge depending on its amount. When a piezo transducer drives oscillation of the upper electrode with this electric field, an AC current is induced. The amplitude $A$ of the $\mathrm{AC}$ current is given by

$A=\frac{a \omega\left(b Q+\varepsilon_{0} S \phi\right)}{d^{2}}$,

where $a, w$ are the amplitude and angular frequency of the upper electrode oscillation, respectively, $b$ is the distance of the liquid surface from the bottom electrode, $Q$ is the total charge on the surface, $\varepsilon_{0}$ is the permittivity of vacuum, $S$ is the surface area of the electrode and $d$ is the gap between the electrodes. In our experiment, $\omega / 2 \pi=880 \mathrm{~Hz}, a=50 \mathrm{~nm}, b=1.5 \mathrm{~mm}, S=4.9 \mathrm{~cm}^{2}$, $\phi=81 \mathrm{~V}$ and $d=3.0 \mathrm{~mm} . A$ is measured by using a current-to-voltage converter and a lock-in amplifier. An advantage of using VCE is that the measured quantity $A$ behaves linearly as a function of $Q$ (Eq. (1)).

Fig. $1[A]$ shows the calibration curve of VCE signal to the number of surface electrons $N_{\mathrm{e}}=Q / e$, where $e$ is the electronic charge. Compared with the Corbino electrode signal calibration curve (Fig. 1 [B]), VCE provides much improved measurements of the surface charge, especially at $N_{\mathrm{e}}>3 \times 10^{8}$ electrons.

The sample cell is connected by a capillary to the $\mathrm{H}_{2}$ dissociator, where $\mathrm{H}$ atoms are produced by pulsed RF glow discharge. $\mathrm{H}$ atoms were introduced to the sample cell, in which the surface electrons had been 


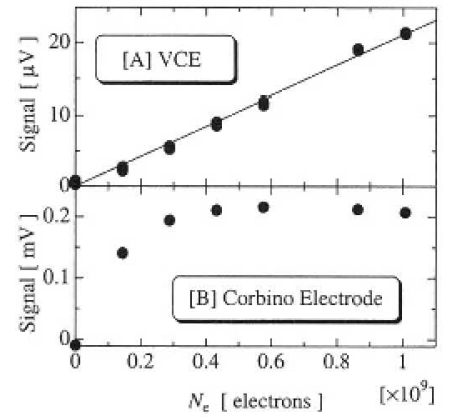

Fig. 1. The signal amplitude calibration as a function of the total surface electron number. [A]: Vibrating capacitor electrometer, [B]: Corbino electrode. For [A], the constant term is subtracted. The linear behavior of VCE signal is a great advantage. The solid line is a linear fit.

prepared in advance, while the total surface charge was measured. We found that the surface charge decreases by the introduction of $\mathrm{H}$ with temperature-dependent rates. Examples of VCE signal evolution are shown in Fig. 2. The filled and open circles denote the decay signals at 0.26 and $0.15 \mathrm{~K}$, respectively. The reduction of the surface charge suggests that $\mathrm{H}^{-}$ion does not stay on the surface but it penetrates into the bulk liquid. Since the kinetic energy of $\mathrm{H}^{-}$is not more than the reaction energy, 0.75 $\mathrm{eV}$, it is not expected to leave the surface to the gas phase against the holding potential $\phi$ of the order of $10^{2} \mathrm{~V}$.

The measured reaction rates are faster at lower temperatures. This means that the reaction takes place in the adsorbed phase mixture of $\mathrm{H}$ and $\mathrm{e}^{-}$, since the surface density of $\mathrm{H}$ is higher at lower temperatures. It is consistent with the claim in our earlier report [1]. However, the

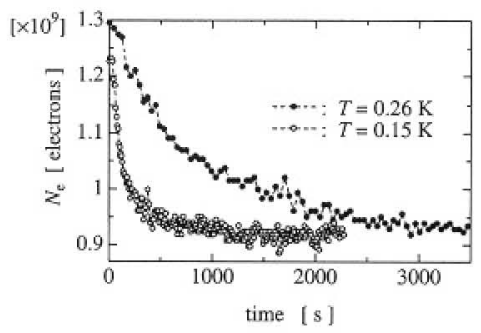

Fig. 2. The number decay curves of surface electrons after the introduction of same amount of $\mathrm{H}$ atoms. The filled and open circles are the data at 0.26 and $0.15 \mathrm{~K}$, respectively.

decay is not expressed as a simple exponential and the assumption we made in our earlier rough analysis that the recombination of $\mathrm{H}, \mathrm{H}+\mathrm{H} \rightarrow \mathrm{H}_{2}$, can be ignored may not be strictly correct. A proper model to explain all the data is required.

\section{Acknowledgements}

This work was partly supported by Grant-in-Aid for Scientific Research from Monbusho and JSPS.

\section{References}

[1] T. Arai, T. Shiino, K. Kono, Proceedings of the 13th International Conference on the Electronic Properties of TwoDimensional Systems (EP2DS), Ottawa, 1999; (special issue) Physica E. 